\title{
Suicide thoughts
}

D Samarasinghe

\section{Abstract}

The unmatched drop in suicides that Sri Lanka achieved around the turn of the century provide lessons for what is useful to try now, as our rates gradually plateau. Looking back over that phase of dramatic reduction in rates provides an opportunity also to examine critically the strategies used in other countries, to reduce suicides. And examining little explored facets of the puzzling phenomenon that suicide is, helps us develop new insights about our own unsuicidal existence.

SL J Psychiatry 2021; 12(2): 1-3
Taking yourself way too seriously leads to suicide. So does extreme self-negation or altruism. Many other personality predispositions act as suicide pulls and pushes. Societal slants too encourage or discourage suicide. Chance events, habits, the nature of some close or not-close-enough relationships, increasing age, isolation, and degree of religious faith are associated with suicide. Many mental illnesses, including forms of substance use, show strong or weak associations with suicide. Causality attributions to such associations are lazily accepted or stubbornly rejected, based mostly on individual bias.

Clear thinking is essential for effective preventive action. It is easy to be distracted by seemingly plausible, but minimally effective measures. Some interventions can lead to unexpected untoward outcomes. Testing new interventions is bedeviled by complex ethical issues and serious practical difficulties, including the problem of assessing impacts due to the relatively rarity of suicide.

We in Sri Lanka and others elsewhere have made progress in reducing suicides through planned interventions, despite the complexity and pitfalls involved. More can be achieved. We should examine critically the things that likely work, and periodically take stock. Subjecting our views to critical scrutiny has benefits.

\section{Some background issues}

First to consider is the ethics of suicide prevention. When invited some quarter century ago to contribute to formulating and implementing a national response to reduce Sri Lanka's then record-high suicide rate, I was initially uncertain whether to accept. What right does someone have to interfere with another's personal decision - even though the resulting action is extreme and, in some instances, undertaken for seemingly trivial reasons.

For psychiatrists, the most powerful argument in favour of a preventive intervention is probably that the wish to kill oneself is not really a free choice, when it is part of a mental illness. We know also that most individuals who survive self-harm later claim that they are relieved they did not die. Our wish to prevent such suicides therefore appears justified. This justification may then be extended to cover all suicides and sometimes veer towards seeing suicidality itself as a sign of mental illness (1).

For me, a more compelling consideration in accepting the invitation to join in the prevention effort was the unmitigated suffering that suicide inflicts on those bereaved as its result. Pain due to a loved one's suicide death is hard even to imagine.

Psychiatry and allied disciplines have substantial contributions to make in this field. But they are only part of the several measures needed to reduce suicides in a population. We must examine carefully whether proposing to expand psychiatry and allied services as a means to reduce suicide can be justified.

\section{Sri Lankan experiences}

It is time to re-examine the systematic actions implemented in Sri Lanka, from 1995, which were followed by a remarkable reduction in suicides. Rates dropped by more than half in the first 10 years of implementation and sustained a further, slower, drop since $(2,3)$. 
The fair number of scientific articles on suicide in Sri Lanka that have been published in the last 20 years is probably due to this unparalleled drop in national suicide rates (4). The initiatives undertaken at the time were driven by the President establishing a group to address suicides, which were then reported to be the highest in the world. The team was provided the means to carry out interventions and was later formalized as a 'Presidential Task Force on Suicide Prevention'. Widespread grass roots implementation of its proposals was ensured through a team of District Development ('Samurdhi') Managers. Two Managers from each District were released for full time work on reducing substance use and suicide, starting from 1995. They were continually trained, and the progress of their activities monitored at regular sessions for more than four years. They had access to the entire national network of Samurdhi Development Officers in their districts - through whom they were able to mobilize widespread action by the citizenry. The authority of the Task Force, as an initiative of the freshly elected President herself, facilitated action from all concerned ministries.

The Task Force, while implementing actions to reduce suicides, was asked next to prepare a formal policy and plan of action as well (5). Lessons derived from ongoing interventions, even prior to the policy being drafted, powerfully influenced the recommendations that were finally included in the action plan. Following formal approval of the policy by the Cabinet of Ministers, the task force was asked to implement the proposals even more vigorously. It reported progress, at intervals, directly to H.E. the President and more frequently to her Advisor on Social Infrastructure, who worked closely with her.

The recommended measures, listed next, were thereby applied widely, in most parts of the country. The role that the lay public could play in implementing recommended interventions was maximized and action was not restricted to bureaucrats and professionals. Engaged communities were provided means to check whether their actions were on track. The main content of activities is listed next.

1. Minimizing easy access to highly lethal means of selfharm was pursued through several means. This intervention was characterized by sceptics as a superficial and insensitive response to the deep anguish of a suicidal person. But widespread actions were steadily generated in an increasing number of settings, mainly rural, to ensure that highly lethal agricultural pesticides were not kept readily or easily accessible. Many rural groups also eradicated freely growing poisonous plants, where these frequently figured in suicide. Such actions were widely implemented through the designated development officers released for this task. When the benefit of the measures began to be noticed in communities that implemented these actions vigorously, the resistance in others diminished.
Steps to prohibit the sale of the most lethal pesticides was instituted, through state action, and eventually put in place after the usual commercially-driven resistances for such measures were gradually overcome.

2. Improving the management of poisoning was addressed actively. It was found that individuals who had swallowed agricultural pesticides were often not dealt with effectively at the nearest local hospital. Some were not dealt with at all, but directed to the next level. Vital immediate management was thereby delayed. Other matters regarding management were also noticed - such as large differences in outcomes among hospitals of similar level. Variations in the attitude and ability of staff was identified as a likely contributor. Remedies were instituted by Provincial and District Health authorities, and instructive innovations shared.

3. Changing how much, and how, the mass media report suicide was a major success. Much effort was expended in steadily winning over the various agencies involved - both public and private - through repeated appeals, discussions, and positive feedback when salutary changes were noticed. Powerful persons and media agencies that were initially resistant, seeing the appeal as an unwarranted interference with media freedom, were gradually won over through sustained coaxing. A few that were obstinately insistent that there was not enough justification to change current practice had to be addressed through other means, such as public pressure. These too were carried out by activists in communities reached through the designated government officers.

4. Changing the social milieu towards discouraging suicide was an innovation that evolved steadily and with constant monitoring of results, given the complex issues involved. The initiative was based on the visible finding that some cultural features, such as those operating in Islamic communities, protected against suicide. The interventions were aimed at engaging more vulnerable communities to establish such protective norms in their settings too. The relevant features of those cultures with extremely low suicide rates were examined, analyzed and shared with others. The common theme noticed was a social invalidation or rejection of suicide as a response to distress or suffering, however serious. Similar negative perceptions were seen to be applied to even the expression of suicidal intention. To help other communities to analyze, understand, and reverse the many factors which led to acceptance of suicide in their communities was difficult. The difficulty was compounded by the need to guard against the process resulting in insensitivity to individuals under dire and desperate circumstances. Progress on both fronts by community groups was impressive. 


\section{Reflections}

A question that arises is whether giving the lead role to psychiatry and allied professions harms or hinders suicide prevention efforts directed at populations. These professions are generally seen as the most appropriate to provide technical leadership in suicide prevention efforts. But expanding individually focused 'mental health' type of interventions did not find a place among the main elements in the policy formulated in Sri Lanka. This was because sufficient evidence in support was found neither in studies published until that time nor through the actions initiated at the inception, while the proposals were being prepared. Given the dramatic and sustained drop of suicide rates seen from the start of our national initiative, we should re-examine the appropriate place, in suicide prevention generally, for psychological interventions directed at individuals.

Quite an unrelated matter that merits reflection is how individuals who subscribe strongly to religious beliefs that forbid suicide still manage to plan and carry out premeditated attempts to end their life. There is much to learn by listening to what individuals who've survived such attempts say about how they reconciled their seriously suicidal act with the opposing religious faith. Psychiatrists are well-placed to explore this.

A further aside to reflect on is what may be called semisuicidal conduct. This ranges from tobacco smoking to holding passionately to views inimical to one's own wellbeing. The underlying drive is generally provided by intense religious, political, or economic convictions. Many fanatical ideologues can be said to be indulging in deliberate and serious self-harm, though they are not classified as suicidal. A different form of partial suicide is the tendency to kill off vital parts of life's panoply in allconsuming preoccupation with activities such as gaming, gambling, meditation, substance use, or the single-minded pursuit of wealth, fame, or power.

An interesting speculation, not entirely idle, is whether animals can commit suicide (6). Most suggestive accounts involve domesticated dogs. The question has potential to instruct. For one, we may discover incontrovertible evidence of such acts that we'd dismiss out of hand. And when we do, many insights may dawn. For example, what does it take to develop a sense of an independent and autonomous self? and how sophisticated, or silly, is the capacity to choose deliberately to destroy that self? We may recognize new reasons as to why some humans are totally incapable of contemplating suicide. As an aside, I have come across an instance where a non-domesticated mammal did deliberately kill itself. 'It' feels like the wrong pronoun for this individual.
Suicide bombers cannot be ignored here. They can teach us not only about extreme human gullibility of the bomber but also about the murky mental depths inhabited by those who recruit, train and explode her or him, to serve causes for which they will not sacrifice their own lives. Their cousins target the rest of us, for less dramatic forms of self-annihilation - through making us blinded zombies that mindlessly echo their every thought.

The final thought is on assisted suicide. Early justifications revolved around relieving physical suffering in certain terminal conditions. Assisting suicide is now beginning to be accepted as a response to all sorts of other seemingly compelling personal reasons - such as avoiding indignities related to decay and disability. Perceiving some circumstances as insufferably humiliating can be the result of taking oneself way too seriously.

D Samarasinghe, formally of the Department of Psychiatry, Faculty of Medicine, University of Colombo, Sri Lanka

Email: samara-singhe@hotmail.com

http://orcid.org/0000-0003-2891-8297

\section{References}

1. Reardon S. Suicidal behaviour is a disease, psychiatrists argue. New Scientist 2013; https://www.newscientist.com/ article/dn23566-suicidal-behaviour-is-a-diseasepsychiatrists-argue/

2. de Silva VA, Hanwella R, Senanayake N. Age and sex specific suicide rates in Sri Lanka from 1995-2011. Sri Lanka Journal of Population Studies 2012; 3(2): 7-11.

3. N Thalagala. Suicide trends in Sri Lanka 1880- 2006; social, demographic, and geographical variations. Journal of the College of Community Physicians of Sri Lanka 2009; 14 (1): 24-32.

4. Rajapakse TN. A review of the changing patterns of suicide and deliberate self-harm in Sri Lanka. Sri Lanka Journal of Psychiatry 2017; 8(1): 3-9.

5. National Policy and Action Plan on Prevention of Suicide 1997. WHO: Country Resources » Sri Lanka https:// www.mindbank.info/item/6096

6. Peña-Guzmán, DM. Can non-human animals commit suicide? Animal Sentience 2017; 20(1): 1-24. 